\title{
Conceptions nouvelles dans la préparation des vaccins et l'usage des vaccinations
}

De la vaccination jennérienne aux nouveaux vaccins déjà obtenus par recombinaison génétique et bientôt par synthèse chimique, cet article fait le point du chemin parcouru ainsi que des perspectives de recherche.

\section{Michel Rey}

Professeur, maladies infectieuses et tropicales. Conseiller de l'OMS

\section{RÉFÉRENCES}

1. Hilleman MR. Newer directions in vaccine development and utilization. $J$ lnfect $D$ is 1985 ; 151, 3 : 407-19.

2. Ajjan N. La Vaccination I. Lyon : Mérieux, 1985: $286 \mathrm{p}$.

3. Rey M. Abrégé des Vaccinations. Paris : Masson, 1980 : $286 \mathrm{p}$.

4. Voller A, Friedman H. New Trends and Developmenls in Vaccines. Lancaster (UK) : MTP Press, 1988 : $323 \mathrm{p}$.

5. Hannoun C. Vaccinations : présent et futur. Reoue du Praticien 1980 ; 30, 15 : 981-5.

\section{ADRESSE}

M. Rey : CHU-Hôtel-Dieu, boulevard L. Malfreyt, 63000 Clermont-Ferrand. $\mathrm{m} / \mathrm{s} n^{\circ} 2$ ool. 3, ftoriar 87

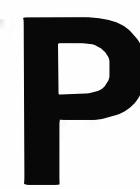

our apprécier l'importance de la révolution des techniques, des concepts et des stratégies qui bouleverse actuellement le domaine des vaccinations et le projette vers l'avenir, il est utile de regarder en arrière et de se remémorer l'histoire des vaccinations humaines dont nous disposons aujourd'hui $[1,2]$.

\section{Le chemin parcouru}

Cette histoire des vaccinations humaines se déroule à notre avis en deux grandes étapes :

(1) La mise en place des premières vaccinations historiques : de la vaccine de Jenner (1796) au bacille de Calmette et Guérin (1924). La vaccination de Jenner s'inspirait du vieux concept asiatique de l'inoculation volontaire de la variole, qui s'était propagée en Europe au XVII' siècle. Découverte occasionnellement par un praticien généraliste, elle utilisait un virus animal naturel (cowpox) apparenté à celui de la variole, tiré des lésions cutanées de génisses infectées.

C'est Pasteur qui inventa les premiers vaccins préparés au laboratoire, vaccins vivants atténués par passages multiples : choléra des poules (1879), charbon (1881), rage (1885), le vaccin rabique étant le premier vaccin atténué appliqué à l'homme. Cette voie conduira à la mise au point du BCG (bacille tuberculeux bovin atténué). Une autre voie est ouverte par Wright en 1892 avec le vaccin typhoïdique : celle des vaccins bactériens tués par la chaleur et le formol, qui aboutira plus tard au vaccin coquelucheux (Madsen 1923, Leslie et Gardner 1933). Ces vaccins de première génération restent très proches des agents infectieux naturels. Ils sont irrégulièrement efficaces (à l'exclusion de la vaccine) et plus ou moins bien tolérés.

Pendant cette même période, le concept de vaccination généralisée se met en place (obligation légale de la vaccination contre la variole en France à partir de 1902, vaccination par le TAB de l'armée française à partir de 1916).

(2) L'avènement des vaccins modernes et la mise au point des stratégies vaccinales : des anatoxines de Ramon (1923-27) au vaccin contre l'hépatite $B$ (1975) et à l'éradication de la variole (1977-80). Les anatoxines diphtérique et tétanique sont les 


\section{RÉFÉRENCES}

6. Mise au point de vaccins - nouvelles approches : memorandum d'une réunion de l'OMS. Bull Org Mond Santé 1985 ; 63, 5 : 851-7.

7. Roitt I. New Trends in Vaccines. London : Academic Press, 1984 : 145 p.

8. Rey M. Vaccinations : présent et avenir. Médecine at Maladies Infectieuses $1985 ; \mathrm{n}^{\circ}$ spécial : 62-5.

9. Recombinant vaccinia viruses or live vectors for vaccine antigens : memorandum from a WHO/USPHS/NIBSC meeting. Bull Org Mond Santé $1985 ; 63,3: 471-7$.

10. Edelman $R$. Vaccine adjuvants. Rev Infect Dis $1980: 2,3: 370-83$.

11. Mc Cullough KC, Langley D. Antiidiotope vaccines : can they exist? Vaccine $1985 ; 3,1: 59-64$.

12. Kennedy R, Melnick J, Dreesman G. Anti-idiotypes et immunité. Pour la Science 1986 ; 107 : 52-63.

13. Levine MM. Vaccines to Present Bacterial Enteric Infections. Proceedings IXth Intern. Congress Infect Paras Diseases. Munich : MMV/Medizin Verlag, 1986 : 106-9.

14. Salk D, Salk J. Vaccinology of poliomyelitis. Vaccine $1984 ; 2,1: 59-74$.

15. Sabin AB. Oral poliovirus vaccine : history of its development and use and current challenge to eliminate poliomyelitis from the world. J Infect Dis 1985 ; 151, 3 : 420-36.

16. Ogra PL, Fishaut $M$, Gallagher $M R$. Viral vaccination via the mucosal route. Rev Infect Dis $1980 ; 2,3$ : 352-69.

17. Robinson A, Irons LI, Ashworth LAE. Pertussis vaccine : present and future. Vaccine $1985 ; 3,1: 11-22$.

18. Maassab HF, Deborde C. Development and characterization of cold-adepted viruses for use as live virus vaccine. Vaccine $1985 ; 3,5$ : 355-69.

19. Principles of malaria vaccine trials : Memorandum from a WHO meeting. Bull. Org Mond Santé $1986 ; 64,2$ : 185-204.

20. Stuart-Harris C. The principles and practice of immunization. Practitioner $1975 ; 215$ : 285-93.

21. L'acceptabilité des vaccinations de l'enfant. Ś́minaire du Centre Intemational de l'Enfance (Paris) $1986: 334 \mathrm{p}$.

22. Contre-indications aux vaccinations (circulaire du 27 déc. 1985). Bulletin Épidémiologique Hebdomalaire 1986 ; 4 : 14-5.

23. Manciaux M. Éthique et vaccinations des enfants. Sciences Sociales et Santé $1984 ; 2,3-4$ : 167-89. chefs de file d'un nouveau type de vaccins inertes, les sous-unités vaccinales, ou fractions antigéniques, qui s'opposent aux vaccins complets et qui vont faire progresser l'efficacité et la tolérance, ainsi que la connaissance de la réponse immunitaire. Avec les anatoxines, les adjuvants de l'immunité vont entrer dans la pratique vaccinale. Les polyosides bactériens capsulaires (Gotschlich 1985) constitueront un nouveau type de sous-unité vaccinale s'appliquant au méningocoque, au pneumocoque, à $\mathrm{Hae}^{-}$ mophilus influenzae $b$.

Cette période est marquée par le prodigieux développement des vaccins viraux qui accompagne les progrès des techniques virologiques : culture sur œuf embryonné permettant la mise au point du vaccin amaril $17 \mathrm{D}$, vivant (Theiler 1937), et du vaccin grippal inactivé (Salk 1937); puis cultures cellulaires in vitro qui conduiront aux vaccins contre la poliomyélite (Salk 1953, Sabin 1956), les oreillons (Smorodintsef 1954), la rougeole (Enders 1958), la rubéole (Meyer et Parkmann 1962). La production industrielle des vaccins viraux va s'améliorer au fur et à mesure de l'évolution des substrats cellulaires : rein de singe, cellules embryonnaires animales, cellules diploïdes humaines, cellules en lignée continue. La lyophilisation permet la conservation prolongée des vaccins vivants. L'avènement du premier vaccin contre l'hépatite B (Maupas, Hilleman 1975-1980), sous-unité virale corpusculaire inerte extraite du plasma de donneurs humains, se place à la charnière de cette période et de l'étape actuelle.

Parallèlement on assiste, au cours de cette deuxième période, au développement des vaccinations généralisées, à la mise en place des calendriers de vaccination des enfants, à l'élaboration de stratégies vaccinales, au lancement de campagnes de vaccinations de masse. La campagne mondiale d'éradication de la variole aboutit en 10 ans (1967-77) à la disparition de ce vieux fléau humain, réalisant le vœu formulé par Jenner dès 1798 (figure 1). En 1975,

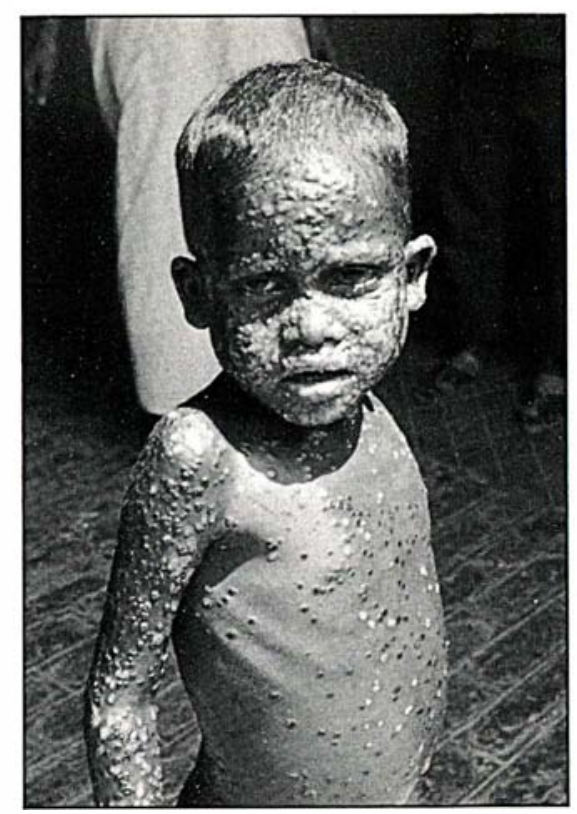

Figure 1. L'un des derniers cas de variolo observé en Inde, à Calcutta, avant l'élimination de l'endémie variolique dans le subcontinent indien (1975). (Cliché M. Rey).

l'OMS lance le programme élargi de vaccination visant l'ensemble des enfants de la planète.

Au terme de ces deux étapes, historique et moderne, nous disposons d'un arsenal bien fourni en vaccins opérationnels [2, 3, 4]. L'application large des plus efficaces d'entre eux a déjà beaucoup contribué au recul de plusieurs maladies infectieuses courantes : diphtérie, tétanos, poliomyélite, coqueluche, rougeole, rubéole. A l'inverse, certains vaccins n'ont joué qu'un rôle mineur dans le contrôle des maladies qu'ils visaient, tels le BCG dans la tuberculose et les vaccins cholérique et typhoïdique.

\section{Vers les vaccins de demain}

Depuis une dizaine d'années, on assiste à une accélération vertigineuse de la recherche, pulsée par 
l'avancement des connaissances en immunologie, en génétique, en biologie moléculaire et par les progrès de la biotechnologie $[1,5$, $6,7]$. Les techniques de production à large échelle des vaccins existants se sont développées, stimulées par l'extension planétaire des vaccinations. Les méthodes de purification et les contrôles d'efficacité et d'innocuité ont progressé : les vaccins d'aujourd'hui sont devenus beaucoup plus sûrs que ceux d'hier. Ils doivent répondre à des normes internationales bien définies. La conservation des vaccins viraux vivants thermolabiles (fièvre jaune, rougeole) a été récemment améliorée. Mais surtout de nouvelles voies sont tracées, dans des laboratoires de recherche fondamentale où vont maintenant s'élaborer les nouvelles générations de vaccins. La possibilité de produire des anticorps monoclonaux à partir d'hybridomes cellulaires a représenté une étape décisive dans cette recherche. Étroitement spécifiques, les anticorps monoclonaux permettent d'identifier avec précision les antigènes intervenant dans la réponse immunitaire protectrice (neutralisation de l'agent infectieux). Ils servent aussi à tester le comportement et l'efficacité des fractions antigéniques à l'étude. Les acquisitions résultant du développement du génie génétique. D’un intérêt considérable, elles apportent de nouveaux outils et de nouvelles techniques de production de vaccins. Elles permettent de contourner certaines difficultés, telle l'impossibilité de cultiver in vitro l'agent infectieux. Les techniques de recombinaison génétique de l'ADN vont intervenir directement dans la production de nouveaux vaccins [7]. Leur principe est d'insérer in vitro dans le matériel génétique d'un virus, d'une bactérie ou d'une cellule, un fragment d'ADN hétérologue capable de modifier la configuration antigénique de toute la descendance (clone) du virus ou du microorganisme initial. Deux voies d'application de cette technique de clonage sont déjà opérationnelles. La première est la $m / s n^{\circ} 2$ ool. 3, ftoria 87 synthèse génétique de fractions antigéniques (sous-unités) par une cellule procaryote (bactérie) ou eucaryote (levure, cellule animale) (voir Lexique $\mathrm{m} / \mathrm{s} n^{\circ} 10$, vol. 2, p. 579). Ce procédé rend possible la production in vitro par le clone recombinant de grandes quantités d'antigène, qu'il suffira ensuite d'extraire des cellules productrices et de purifier. La quantité et la qualité de l'antigène ainsi synthétisé sont généralement supérieures quand on utilise une cellule eucaryote (levure ou cellule animale). C'est par cette technique (figure 2) que sera produite la deuxième génération de vaccins contre l'hépatite $B$, sur le point d'être commercialisée [1]. Parmi les nombreuses autres applications, à l'étude, de ce type de synthèse de sous-unités vaccinales, citons le virus de l'herpès, celui de la grippe, le paludisme.

La seconde de ces voies est l'élaboration de vaccins vivants recombinants. Une première approche a consisté à obtenir, par double infection d'une culture cellulaire in vitro, un hybride de deux virus de la même espèce, mais de types antigéniques différents ( $p a r$ exemple deux virus grippaux $\mathrm{A}, \mathrm{H} 1 \mathrm{~N} 1$

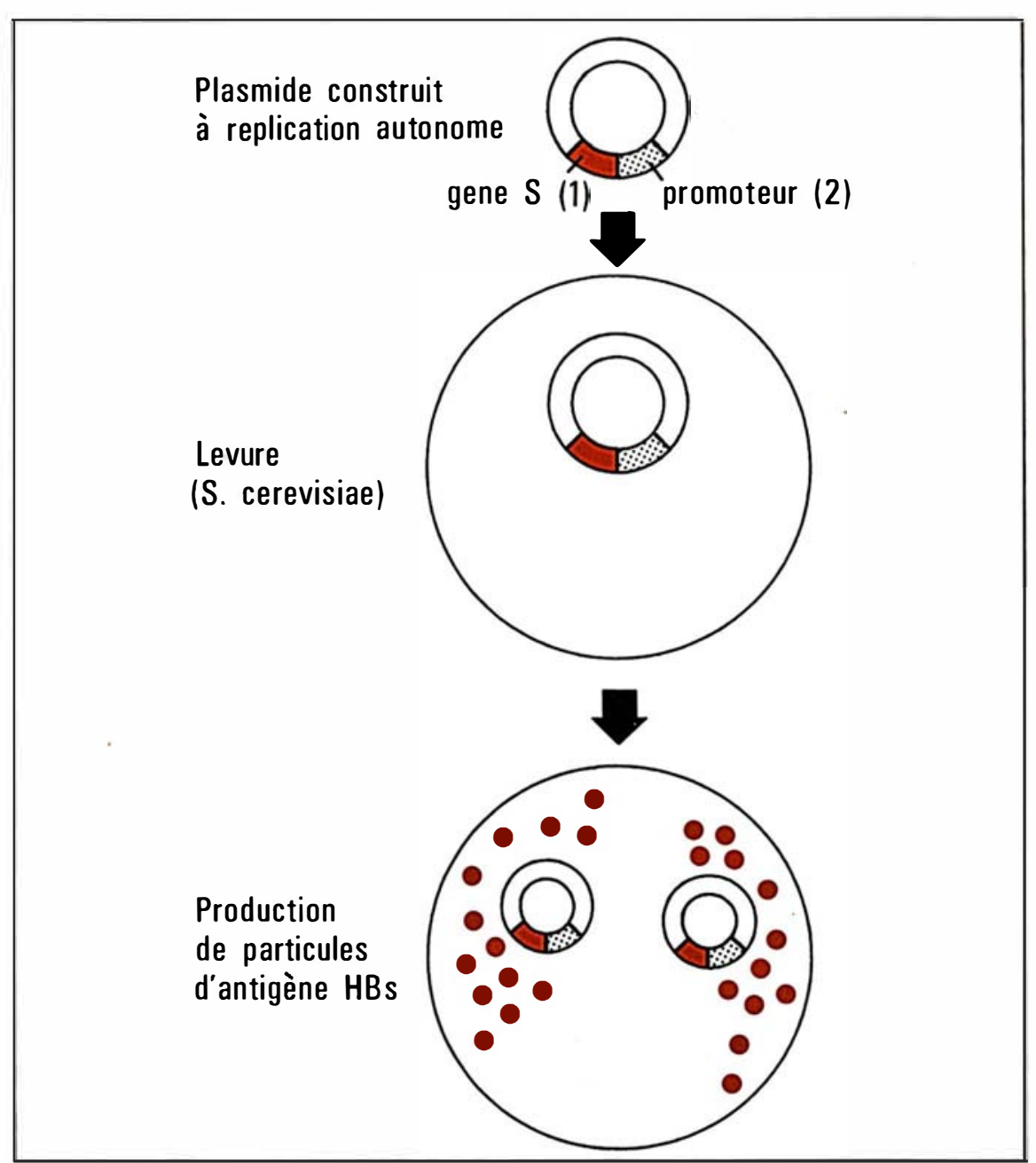

Figure 2. Schéma do la production sur lovure d'un vaccin recombinant contre I'hépatite B. (1) Gène du virus HB codant la production d'antigène HBs; (2) promoteur alcool-déshydrogénase I de la levure. 




Figure 3. Hybridation d'un virus de la vaccine par recombinaison de I'ADN in vivo, en vue de la préparation d'un vaccin vivant recombinant (inspiré de G. Smith, dans l'ouvrage de Roitt [7]).

et H3N2, dont l'hybridation peut aboutir à un " réassortiment " du type $\mathrm{H} 12 \mathrm{~N} 2$ ou $\mathrm{H} 3 \mathrm{~N} 1)$. La recombinaison génétique de l'ADN permet d'aller plus loin, en insérant dans le génome d'un virus porteur (ou plus rarement dans une bactérie) un fragment d'ADN de l'agent infectieux visà-vis duquel on veut obtenir un pourrait être particulièrement intéressant pour les pays en développement. La troisième génération de vaccins contre l'hépatite $B$ devrait logiquement appartenir à cette catégorie de vaccins vivants recombinants.

Vers la synthèse chimique des vaccins. L'identification précise de déterminants antigéniques de petit poids moléculaire (oligopeptides, glycoprotéines) permet leur synthèse chimique. On a déjà réalisé la synthèse d'une protéine virale de la mosaïque du tabac, de l'antigène carcino-embryonnaire, de la toxine diphtérique, de la protéine $\mathbf{M}$ du streptocoque $A$, et de quelques antigènes viraux (fièvre aphteuse, antigène $\mathrm{HBs}$, hémagglutinine du virus grippal). Ces antigènes synthétiques sont très spécifiques, leur spécificité étant liée à leur structure chimique et particulièrement à leur configuration spatiale. Mais ils sont faiblement antigéniques : pour obtenir une réponse anticorps il faut les conjuguer avec une grosse molécule protéique " porteuse " et avec un adjuvant naturel ou synthétique (muramyldipeptide ou MDP) $[7,10]$. Les perspectives ouvertes par ce genre de recherche débouchent sur un nouveau type de vaccin, entièrement artificiel et synthétique et théoriquement plus sûr. Elles étendent le domaine des vaccinations sur des objectifs non infectieux : reproduction, cancer. Déjà, on a pu réaliser une castration immunologique de l'animal par administration d'un vaccin hormonal synthétique (LHRH + MDP). Toutefois, les problèmes de production à grande échelle de tels vaccins et de faisabilité de telles vaccinations sont encore loin d'être résolus.

Vaccins anti-idiotypes. Il a été démontré $[7,11,12]$ que l'inoculation à l'animal d'un anticorps dirigé contre un antigène donné peut induire la production d'antianticorps dont certains sites de liaison ont une configuration spatiale analogue à celle des épitopes de l'antigène initial (figure 4). Cet auto-anticorps (anti-idiotype) peut être utilisé comme un vaccin, la synthèse d'un antigène vaccinant (figure 3). C'est le virus de la pluse qui s'annonce comme plus prometteur des virus poreurs [9] : il est facile à produire, à manipuler, à inoculer. Encore faudrait-il le débarrasser de tout risque d'effet indésirable. Ainsi s'annonce un nouveau type de vaccins vivants, dont le faible coût de production et d'administration 
fonctionnant comme un antigène "suppléant ", pour induire une réponse immunitaire active. Cette réponse est éventuellement protectrice vis-à-vis de l'antigène initial. Cette approche originale pourrait conduire à l'élaboration de vaccins très particuliers constitués d'anticorps (en principe hétérologues). Ces vaccins, encore hypothétiques, pourraient s'appliquer plus particulièrement à des situations dans lesquelles la production ou l'inoculation d'un antigène vaccinant est irréalisable. Non seulement ce projet aboutirait à de nouvelles vaccinations anti-infectieuses, mais aussi il pourrait ouvrir la voie à des vaccinations anti-cancéreuses.

\section{Nouvelles cibles et choix des armes}

L'élargissement du domaine des vaccinations. Les premiers vaccins historiques ont été dirigés vers les grandes priorités infectieuses de l'époque, dans les limites des moyens technologiques disponibles. Il s'agissait d'endémoépidémies meurtrières, qui se répartissent en trois groupes auxquels on s'est successivement attaqué avec un succès assez inégal : (a) les maladies "pestilentielles " (variole, choléra, peste, typhus, fièvre jaune) et autres fléaux de l'humanité (rage, tuberculose, grippe); (b) les maladies infectieuses qui décimaient les armées en campagne (variole, fièvre typhoïde, tétanos) et qui ont suscité assez tôt une réglementation vaccinale propre aux militaires ; (c) les maladies infectieuses meurtrières ou invalidantes des enfants (diphtérie, coqueluche, poliomyélite).

Grâce aux avancées technologiques des 25 dernières années, de nouveaux secteurs de la pathologie infectieuse ont pu être atteints par les vaccinations. Les recherches en cours font espérer des vaccins contre la plupart des maladies infectieuses. Ainsi peuton envisager, dans un avenir plus ou moins proche, l'entrée en lice des vaccinations dans la lutte contre les infections entériques (en améliorant les vaccins contre le $m / s n^{\circ} 2$ ool. 3, ftoricr 87

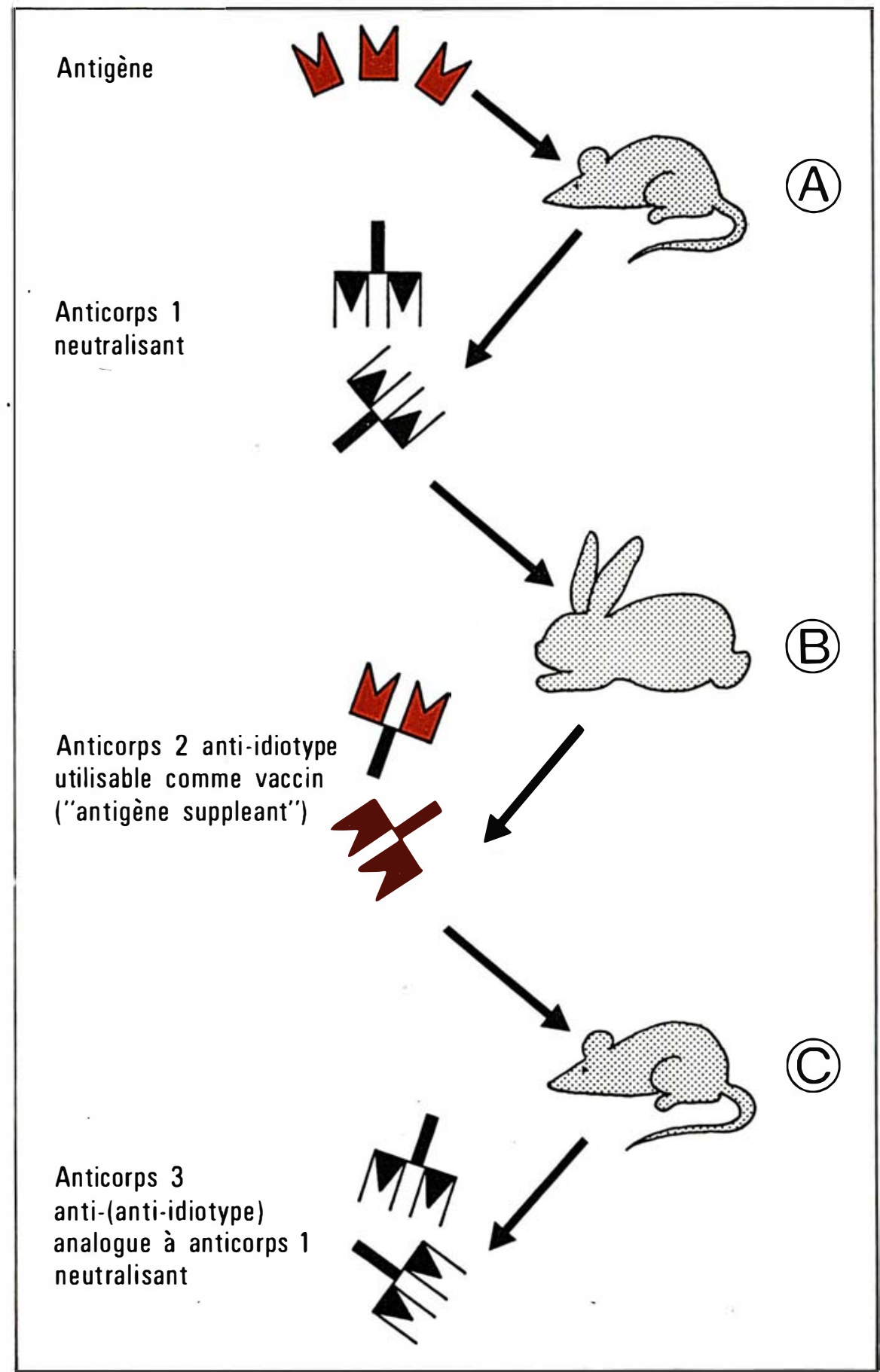

Figure $\Delta_{r}$. Vaccin anti-idiotypo : préparation et application. Les anti-anticorps produits par l'animal $B$ sont utilisés comme un vaccin susceptible d'induire une protection immunitaire active chez l'animal $C$. 
choléra, la fièvre typhoïde, en introduisant des vaccins contre la dysenterie bacillaire, le colibacille entérotoxinogène, les rotavirus) [12], contre les infections respiratoires bactériennes et virales, contre les arboviroses (dengue), contre la lèpre, contre le paludisme et quelques autres parasitoses, contre le SIDA.

Mais le changement d'objectif le plus notable est le développement des vaccinations dans des domaines infectieux où le risque de mort ou d'infirmité grave est très atténué, au moins dans les pays à niveau de vie élevé. Vis-à-vis des infections des enfants, nous disposons déjà de vaccins contre la rougeole, la rubéole, les oreillons (le triple vaccin associé contre ces trois infections a déjà largement fait ses preuves), la varicelle, la méningite à $H$.influenzae. Nous aurons probablement un jour des vaccins contre les virus respiratoires (parainfluenzae, adénovirus, $v$. respiratoire syncitial), l'hépatite $\mathrm{A}$, la mononucléose infectieuse. Deux autres domaines vont se développer : celui des maladies vénériennes (gonococcie, syphillis, herpès, chlamydia) et celui des infections néonatales (aux vaccinations déjà opérationnelles comme le tétanos et la rubéole pourraient s'ajouter des vaccinations contre le streptocoque $\mathrm{B}$, le cytomégalovirus, le toxoplasme). A l'exception des vaccinations contre la rougeole et le tétanos néonatal, fléaux meurtriers du Tiers-Monde, les autres vaccinations entrant dans ces catégories ne se justifient et ne sont réalisables, avant longtemps, que dans les pays développés. Leur justification repose sur des évaluations de Santé Publique, et sur des arguments autant économiques (rentabilité) qu'humains $[1,6]$.

Enfin les vaccinations vont sortir du cadre des infections. Des vaccins contraceptifs sont en vue. Nous disposons déjà d'un vaccin anticancéreux, le vaccin contre l'hépatite $B$ (qui protège en principe contre le cancer primitif du foie). Ce vaccin ouvre la voie à dont la première génération sera dirigée contre les infections virales oncogènes.

Quel type de vaccin choisir? Quelques questions en suspens. L'élargissement actuel de l'arsenal vaccinal multiplie les possibilités de choix entre plusieurs types de vaccins. Lorsque ceux-ci ont déjà été appliqués, on tient compte des performances réellement observées. En fait, pour tout vaccin " à choix multiple" la décision dépend de nombreuses contraintes : efficacité, tolérance, "faisabilité " (production à grande échelle, conservation, administration, atteinte de la population cible) et coût. Parmi les nouveaux modèles de vaccins expérimentaux qui nous sont proposés, combien rempliront ces conditions indispensables pour le passage au stade opérationnel ?

- Une première question se pose : vaccin vivant ou vaccin inactivé ? Les vaccins vivants ont de nombreux avantages : ils reproduisent assez fidèlement l'immunisation naturelle, ils sont assez faciles à produire et peu onéreux, ils sont faciles à administrer (en principe une dose unique est suffisante). Mais ils ne sont pas toujours dénués de risques infectieux (réactions, complications) et on peut s'interroger sur la stabilité et le devenir dans l'organisme de certains virus. Les vaccins inactivés ou inertes sont en principe plus sûrs, exempts de tout risque infectieux (mais parfois sensibilisants) ; cependant ils sont souvent plus coûteux, ils nécessitent plusieurs inoculations et des rappels. La compétition entre ces deux types de vaccins est bien illustrée par l'exemple de la poliomyélite vis-à-vis de laquelle le vaccin inactivé injectable de J. Salk et le vaccin vivant oral de A. Sabin s'affrontent plus que jamais [14, 15]. Les deux vaccins se sont montrés capables d'éliminer la poliomyélite dans les pays industrialisés. Le vaccin vivant, de loin le plus utilisé dans le monde jusqu'à présent, est confronté à deux problèmes : l'émergence d'accidents neurologiques (rares) chez les vaccinés et leur entou- rage, expliqués par un retour à la neurovirulence, et un échec relatif de son efficacité épidémiologique dans les pays chauds. Le nouveau vaccin inactivé renforcé proposé par J. Salk paraît résoudre ces deux problèmes ; son seul inconvénient est son coût, encore trop élevé pour permettre sa diffusion large dans les pays en développement. Même si l'on assiste actuellement à la multiplication de nouveaux vaccins inertes (fractions antigéniques), les vaccins vivants, particulièrement adaptés aux vaccinations de masse, devrait connaître un nouveau développement avec l'essor des vaccins génétiquement recombinés.

- Une deuxième question concerne la voie d'inoculation : muqueuse ou parentérale? Ce dilemme recoupe partiellement le précédent. La voie muqueuse, voie naturelle de l'infection, a toujours paru à juste titre particulièrement appropriée à l'immunisation, qu'il s'agisse de la voie orale pour vacciner contre les infections à porte d'entrée digestive, ou de la voie nasale (ou conjonctivale) pour vacciner contre les infections respiratoires. La commodité de l'inoculation se double d'un avantage immunologique et épidémiologique : la protection immunitaire, renforcée par une immunité locale de surface, s'opposerait plus efficacement au portage et à la dissémination de l'agent infectieux naturel [16]. Mais les faits n'ont pas toujours confirmé ces avantages théoriques. Les taux d'échec de cette voie d'inoculation sont souvent élevés, expliqués par des défenses naturelles qui s'opposent à la " prise " du vaccin. Il faut trois prises orales successives (davantage dans les pays chauds) de vaccin poliomyélitique pour obtenir $95 \%$ de protection. Il faut aussi plusieurs prises orales du nouveau vaccin typhoïdique vivant (souche Ty21a) pour obtenir une réponse, d'ailleurs inégale d'une étude à l'autre. Cependant, la voie muqueuse (orale ou nasale) continue à faire l'objet de recherches, tant pour les vaccins inertes que pour les vaccins vivants. 
- Le renouvellement des vaccins classiques soulève également bien des problèmes. Quelles que soient les imperfections de certains d'entre eux, et quel que soit l'intérêt des nouvelles solutions proposées par les chercheurs, le remplacement des vieux vaccins par des vaccins modernes s'avère long et difficile. Si l'on a réussi à remplacer les vaccins antirabiques classiques, peu satisfaisants, par des vaccins produits sur cultures cellulaires, efficaces et très sûrs, ces nouveaux vaccins coûtent malheureusement trop cher pour être à la portée des pays pauvres. Si l'on a réussi à réaliser la synthèse chimique de la toxine diphtérique, on voit mal comment ce vaccin synthétique conjugué, très élaboré, pourrait remplacer aujourd'hui l'anatoxine naturelle, efficace, facile à produire et très bon marché, compte tenu des énormes besoins mondiaux. Depuis longtemps on cherche à remplacer deux vaccins bactériens entériques discutables, le vaccin cholérique et le $\mathrm{TAB}$ : parmi les nombreux choix proposés (vaccins vivants ou sous-unités inertes, vaccins oraux ou injectables), aucun vaccin n'a encore fait la preuve sur le terrain de son efficacité et de sa "faisabilité ". Deux problèmes occupent l'actualité : (a) Le remplacement du vaccin coquelucheux classique (cellulaire) par un vaccin acellulaire [17]. Le choix s'est porté sur l'association de deux antigènes protecteurs, l'hémagglutinine filamenteuse (FHA) et le facteur favorisant la lymphocytose (LPF), produits par extraction et débarrassés au maximum de l'endotoxine réactogène (LPS). Ce vaccin est expérimenté à grande échelle au Japon et en Suède. Il paraît aussi efficace, et moins réactogène, que le vaccin classique qu'il doit remplacer. (b) Le remplacement du vaccin grippal, qui pose un problème très particulier : ce vaccin doit être capable de faire face rapidement à la prochaine pandémie, résultant d'une mutation de ce virus particulière. ment instable. Parmi les solutions proposées, on s'intéresse particulièrement à un vaccin vivant nasal, hybride obtenu par réassortiment génétique, contenant les antigènes de surface (hémagglutinine et neuraminidase) apparentés au nouveau mutant, et atténué par adaptation au froid [18]. Néanmoins, les problèmes opérationnels difficiles posés en situation épidémique par une telle vaccination sont loin d'être encore résolus.

- Enfin, l'avenement des vaccins parasitaires est en vue et suscite beaucoup d'espoirs, mais se heurte à des obstacles considérables. Les parasites élaborent de très nombreux antigènes, différents d'un stade à l'autre de leur cycle biologique. Il faut déceler et choisir des fractions antigéniques susceptibles d'induire une protection.

Le paludisme, qui représente un enjeu mondial prioritaire, fait l'objet de nombreuses recherches. Trois types d'antigènes sont en compétition, correspondant à trois étapes du cycle parasitaire chez l'homme ; l'effet protecteur attendu de chacun d'entre eux aurait un impact épidémiologique différent, débouchant sur des stratégies différentes [19]. Un vaccin antisporozoïtes éviterait la contamination de l'homme par le moustique vecteur, un vaccin antimérozoïtes s'opposerait aux manifestations cliniques, un vaccin antigamétocytocytes couperait le cycle épidémiologique en empêchant l'infestation du moustique. En fait, le vaccin idéal devrait probablement contenir les trois types d'antigènes, dans la mesure où l'on pourrait les insérer par recombinaison génétique de l'ADN sur un virus porteur facile à administrer. Mais il faudra encore bien du temps avant de pouvoir disposer d'un vaccin opérationnel et disponible à grande échelle.

\section{Évolution des stratégies et des contraintes}

Les nouvelles stratégies. Pendant longtemps les vaccinations ont été perçues et appliquées comme des mesures de protection individuelle. Leur intérêt pour la collectivité (contrôle des épidémies, réduction des endémies) n'était guère perçu que par les autorités sanitaires. Les progrès de l'épidémiologie et quelques expériences récentes (éradication mondiale de la variole, élimination de la diphtérie et de la poliomyélite dans la plupart des pays industrialisés, tentatives d'élimination de la rougeole) ont jeté les bases de stratégies vaccinales d'élimination. Ces stratégies concernent quelques maladies infectieuses universelles très répandues, théoriquement éradicables dans la mesure où elles répondent à certaines conditions : agent infectieux unique et stable, réservoir exclusivement humain, pas de portage prolongé de virus, immunité acquise solide et, bien entendu, vaccin efficace et facile à administrer. Après la variole, les candidats actuellement en lice sont la poliomyélite, la rougeole, la rubéole, les oreillons. La première étape stratégique est l'élimination nationale ou régionale (avec interruption de la transmission autochtone), l'objectif ultime étant l'éradication mondiale [20].

A la suite des Etats-Unis, l'Europe s'est lancée, en ordre encore dispersé, dans une politique d'élimination de la rougeole et, dans certains pays, de la rubéole et des oreillons. Cette politique impose une stratégie vaccinale beaucoup plus contraignante et difficile à appliquer que pour les vaccinations classiques. En effet, il s'avère que l'élimination de ces infections ne peut être obtenue que lorsque l'immunité de groupe des enfants est très élevée, dépassant $90 \%$ (figure 5, voir page suivante); ceci impose, compte tenu des quelques échecs de vaccination, une couverture vaccinale d'au moins $95 \%$. Celle-ci est d'autant plus difficile à atteindre que la population est insuffisamment motivée, du fait de la bénignité habituelle de telles infections. Dans cette situation, l'intérêt collectif, parfaitement démontré par des études coût-avantages, paraît l'emporter sur l'intérêt individuel. Or, la réussite du programme repose sur l'adhésion quasi générale de la population... 
et des professionnels de la santé ; elle nécessite un effort soutenu d'information et de sensibilisation [21].

Le programme élargi de vaccination lancé par l'OMS en 1975 dans les pays en développement limite actuellement ses objectifs au contrôle des six maladies-cible : diphtérie, poliomyélite, coqueluche, rougeole, tétanos, tuberculose. Là encore, une des difficultés majeures est d'obtenir une couverture vaccinale satisfaisante $(\geqslant 80 \%)$, objectif difficile à atteindre dans des populations sous-équipées et peu informées. Le choix de la meilleure stratégie est encore loin d'être établi. La vaccination de routine des enfants dans les centres de santé est souvent décevante. La vaccination par équipes mobiles est la seule stratégie possible dans les zones rurales les plus défavorisées. Certains pays (Brésil, Colombie) ont même lancé de grandes campa- gnes nationales annuelles de vaccination de masse, mobilisant tout le pays.

Les calendriers de vaccination des enfants sont en cours de modification. Pour qu'ils soient bien appliqués, ils doivent être simplifiés au maximum ; la plupart des vaccins peuvent être associés entre eux, voire combinés, le nombre de séances de vaccination peut être réduit à quatre, voire à trois au cours des deux premières années. Le délai de deux mois entre les deux premières inoculations est immunologiquement préférable à celui d'un mois. On envisage d'avancer l'âge de la première inoculation (actuellement deux à trois mois) ; en effet, la possibilité de commencer la vaccination dès les premiers jours de la vie (avec d'autres vaccins que le BCG) a été démontrée pour l'hépatite $\mathrm{B}$, et a commencé d'être appliquée pour la poliomyélite (maladies à transmission très précoce dans les pays chauds). A côté des vaccinations généralisées, on voit se multiplier des vaccinations à usage restreint, limitées à des groupes exposés à des risques personnels, géographiques ou professionnels. Les vaccinations " à la carte ", adaptées à chaque cas particulier, sont appelées à se développer.

Le renforcement des contraintes. Les exigences de qualité et de sécurité s'accroissent à juste titre. Mais les normes de qualité définies par l'OMS ne sont correctement appliquées que dans les laboratoires producteurs bien équipés. Beaucoup de vaccins distribués actuellement dans le monde ne répondent pas encore aux normes internationales.

Une innocuité quasi parfaite des vaccins modernes est requise. Les réactions vaccinales, parfois inévitables, doivent être distinguées des accidents graves, qui sont devenus rarissimes. Le refus des risques

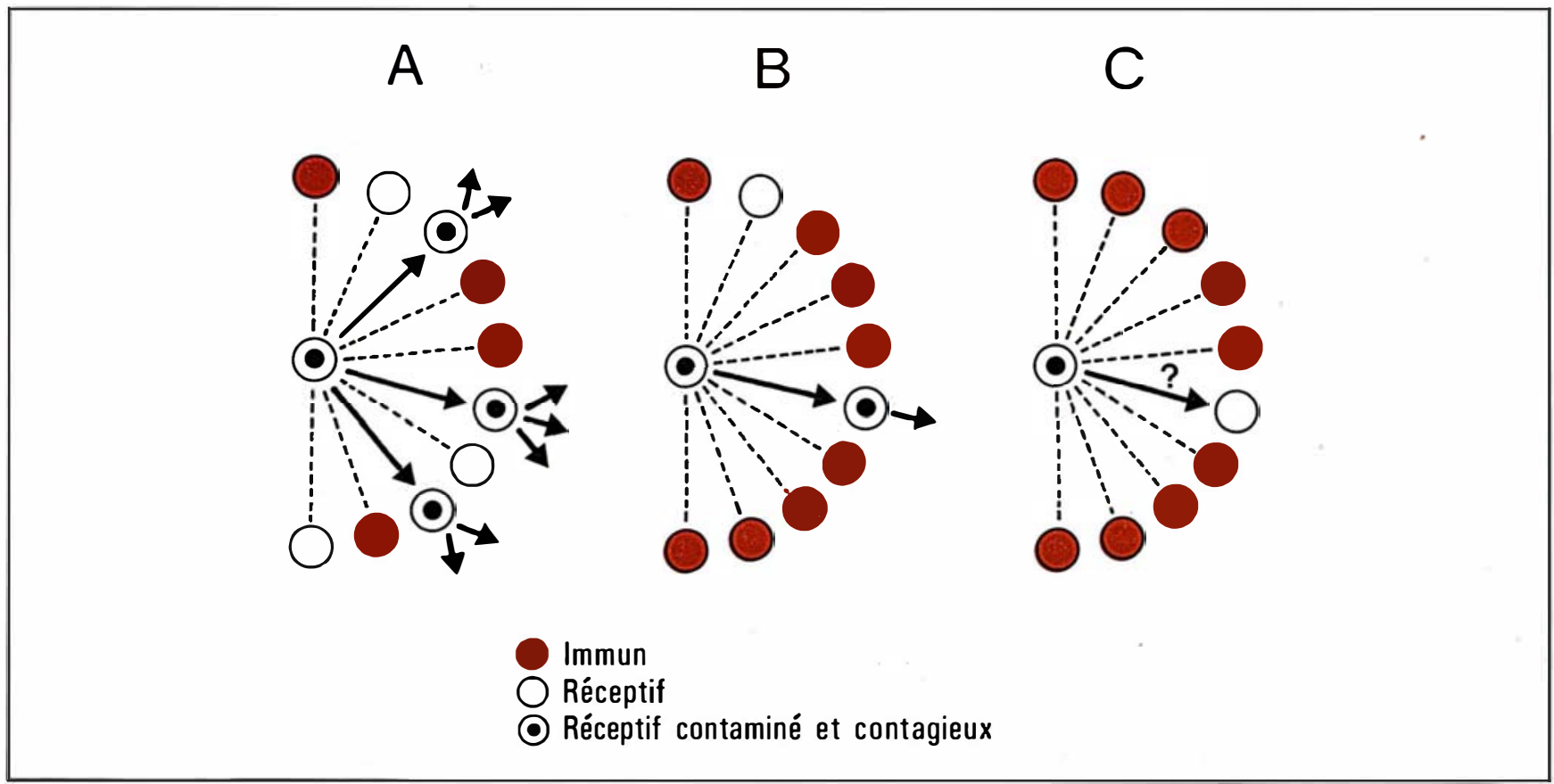

Figure 5. Relations entre l'ímmunité de groupe (pouvant résulter de la couverture vaccinale) et la propagation d'uno infection dans la communauté. IOn a pris comme exemple une maladie infectieuse dont le taux d'attaque est de $50 \%$ : sur deux sujets exposés, un seul est infecté.)

A. immunité de groupe $=40 \%$ : propagation épidémique

B. immunité de groupe $=80 \%$ : persistance d'une endémie faible

C. immunité de groupe $=90 \%$ : élimination possible. 
vaccinaux se radicalise au fur et à mesure que les maladies infectieuses visées perdent de leur gravité. Ainsi, les accidents attribués au vaccin coquelucheux ont sévèrement compromis l'acceptabilité de ce vaccin dans certains pays d'Europe et incitent à remplacer le vaccin classique par un vaccin moderne acellulaire. En fait, l'imputabilité d'un accident à une vaccination est difficile à établir ponctuellement : une simple succession chronologique des événements ne suffit pas à démontrer une relation de cause à effet [3]. C'est par de vastes études épidémiologiques, portant sur des millions de vaccinés, que l'on peut savoir si une vaccination est responsable ou non d'accidents devenus rares : c'est ainsi que la vaccination contre la rougeole a été reconnue exempte de risque d'encéphalite post-vaccinale, la fréquence observée d'un cas pour un million de vaccinés étant celle des encéphalites idiopathiques de la population infantile témoin. Le problème posé par le rôle des vaccinations dans la mort subite du nourrisson est analogue : il ne pourra être résolu que par une démarche épidémiologique. La pharmacovigilance des vaccinations se met en place.

Les contre-indications vaccinales que l'on avait autrefois multipliées par prudence, sont partiellement remises en question et simplifiées [22]. Là encore, c'est par des études épidémiologiques que l'on s'efforce d'établir la justification des contre-indications. Plusieurs d'entre elles se sont avérées non fondées : protéinurie, asthme, diabète. La grossesse a longtemps été exclue des vaccinations par vaccins viraux vivants : cette contreindication est remise en question par le constat de l'absence d'accidents fotaux après vaccination rubéolique de la femme enceinte. Les déficiences immunitaires sont aussi en principe une contreindication des vaccins vivants : mais le vaccin contre la varicelle, surtout appliqué aux enfants leucémiques, remet en cause cette contre-indication.

L'application à l'homme des nou$m / s n^{\circ} 2$ vol. 3, févriar 87 veaux vaccins passe par des études expérimentales dont les normes scientifiques et éthiques sont de mieux en mieux définies. Ces études prospectives, visant à évaluer l'efficacité et l'innocuité des vaccins, sont longues et coûteuses. Il en résulte que le délai entre l'élaboration d'un nouveau vaccin au laboratoire et son application courante s'allonge de plus en plus : plusieurs années sont nécessaires pour l'expérimentation humaine et la mise au point d'une production industrielle. Ces exigences légitimes accroissent le coût des nouveaux vaccins.

Les problèmes éthiques, juridiques et économiques soulevés par l'application des vaccinations sont à l'ordre du jour [3, 21, 23]. Peuton contraindre les individus à subir des vaccinations reconnues utiles à la collectivité ? Les obligations légales sont remplacées dans certains pays par des contraintes administratives difficiles à contourner. La loi de quelques pays admet le refus vaccinal pour raisons philosophiques ou religieuses. Mais ce libéralisme est-il compatible avec la réussite de politiques vaccinales d'élimination, qui nécessite des couvertures vaccinales proches de $100 \%$ ?

Enfin, qui doit payer les vaccinations? Quand l'intérêt de la collectivité est en jeu, il paraît légitime que celle-ci assume l'essentiel des coûts, y compris ceux des éventuels accidents vaccinaux quand aucune faute n'a été commise ni par le producteur ni par le vaccinateur.

En conclusion, l'avancement explosif des recherches actuelles ouvre des perspectives extraordinaires de développement des vaccinations. Mais les difficultés de production et de distribution, les obstacles humains et financiers, ralentissent de plus en plus l'application des nouveaux vaccins. Quoi qu'il en soit, si précieuses soient-elles, les vaccinations d'aujourd'hui et de demain ne représentent qu'une part de la prévention des maladies infectieuses, leur réussite étant conditionnée par leur association à d'autres mesures préventives

\section{Summary}

The accelerated development of knowledge in immunology, molecular biology, biotechnology, is leading today to an unprecedented multiplication of research orientations and of human and animal potential vaccines. The immunization field is spreading not only to most infections but also to cancer and reproduction. New types of vaccines are emerging thanks to the development of genetic engineering, in particular to the DNA recombinant techniques (hybrid live vaccines, genetic synthesis of inert subunits). Vaccines exclusively produced by chemical synthesis are within sight. Such a technological revolution is in contrast with the difficulties encountered in the application of vaccinations : modern vaccines are more and more sophisticated and costly, their industrial production can be difficult, their generalized application stumbles against a great number of logistic, human and economic obstacles. In spite of these difficulties, immunization will probably play an increasing part in the control of infectious diseases in the future. The example of smallpox eradication is stimulating. But it can be expected that the elimination of other major infectious diseases will be more difficult.

\section{TIRÉS A PART}

M. Rey : CHU-Hôtel-Dieu, boulevard L. Malfreyt, 63000 Clermont-Ferrand. 